\section{Asymmetry of masking between noise and tone*}

\author{
RHONA P. HELLMAN
}

Harvard University, Cambridge, Massachusetts 02138

A pure tone was used to mask narrow and wide bands of noise centered on the frequency of the tone. In a given experimental session, the sound-pressure level (SPL) of the tone was held constant and loudness balances were obtained between a masked and unmasked noise band of equal width. These results are compared to earlier measures of the partial masking of tone by noise. The comparison shows that noise masks a tone more effectively than the tone masks the noise. Although the effect of the tone on a critical band of noise is greater than its effect on either an octave-band noise or wide-band noise, it is considerably smaller than the effect of the noise on the tone. Decreasing the noise bandwidth still further to a subcritical width reduces the asymmetry of masking somewhat, but a difference at high intensities of about $20 \mathrm{~dB}$ between the masking effects of an equally intense noise and tone remains. Whether the masker is a tone or noise, masking ceases when the effective energy of the masked and masking stimuli is the same.

A previous study (Hellman, 1970) showed that, when the spectrum level of the noise is held constant, the loudness of a $1,000-\mathrm{Hz}$ tone below a sound-pressure level (SPL) of about $30 \mathrm{~dB}$ is reduced equally by narrowand wide-band noise. However, at higher SPLs, the steepness of the masked loudness function depends on the spread of excitation of the noise. When the bandwidth of the noise is confined to a critical band, the reduction in loudness of the tone is less and therefore the masked loudness function is steeper than it is for broad-band masking. These findings are consistent with earlier assumptions of Zwicker (1963), coupled at near-threshold intensities with the considerations of Zwislocki (1965). Yet, measures of the partial masking of tone by noise leave unanswered the perplexing problem concerning the masking effect of the tone on the noise.

The question of mutual masking between noise and tone has arisen before (Zwicker, 1963; Stevens \& Guirao, 1967), but little is actually known about the partial masking produced by the tone on the noise. Only Zwicker (1963) seems to have measured the reduction in loudness of a noise masked by a tone at the center frequency of the noise band. His data, however, do notprovide information about the growth of loudness of the masked noise as a function of its intensity and bandwidth. Moreover,

\footnotetext{
*This research was supported by NIH Grant NS-02974 (Laboratory of Psychophysics Report PPR-371). The author wishes to thank Professor Bertram Scharf for his constructive criticism of the manuscript and for making unpublished data available and Geraldine Stevens for her assistance with the drafting of the figures.
}

the available data suggest that, although a 1,000- $\mathrm{Hz}$ tone and a critical band of noise of equal intensity are equally loud, the tone masks the noise less than the noise masks the tone. This result appears puzzling.

The present study was undertaken problem of mutual masking when both the masked and masking stimuli are centered on the same sound frequency. Measures of partial and complete masking produced by the tone on the noise were obtained for subcritical, critical, and supercritical bands of noise.

\section{GENERAL PROCEDURE}

The usual roles of noise as masker and tone as signal were simply inverted. For the measurements described in Section II-A, a $1,000-\mathrm{Hz}$ tone masked a narrow-band noise $925-1,080 \mathrm{~Hz}$ wide, an octave-band noise $600-1,200 \mathrm{~Hz}$ wide, and a broad-band noise $75-9,600 \mathrm{~Hz}$ wide. For the measurements described in Section II-B, a 1,400-Hz tone masked narrow bands of noise $1,280-1,480 \mathrm{~Hz}$ wide and 1,350-1,450 $\mathrm{Hz}$ wide. As in an earlier study (Hellman, 1970), the temporal sequence of the masked and unmasked stimuli was the same as that used by Stevens and Guirao (1967), except that the time delay between the offset of the masking stimulus and the onset of the unmasked stimulus was increased from 400 to $800 \mathrm{msec}$. The narrow bands of noise were obtained by passing a wide-band Gaussian noise through bandpass filters that resembled the ones used by Zwicker, Flottorp, and Stevens (1957). Beyond the half-power points, the attenuation of the $925-1,080-\mathrm{Hz}$ filter reached $30 \mathrm{~dB}$ at 760 and $1,300 \mathrm{~Hz}$. The attenuation of the $1,280-1,480-\mathrm{Hz}$ filter reached $30 \mathrm{~dB}$ at in order to examine more closely the
950 and $1,950 \mathrm{~Hz}$ and that of the $1,350-1,450-\mathrm{Hz}$ filter reached $30 \mathrm{~dB}$ at 1,100 and $1,800 \mathrm{~Hz}$. The octave-band noise was obtained with a continuously variable constant-K network Allison filter (Model No. 2AB). Beyond the half-power points, the filter attenuation reached $30 \mathrm{~dB}$ at 360 and $2,300 \mathrm{~Hz}$.

Masked and unmasked noise bands of equal width were matched in loudness by the method of adjustment. The adjustments were made with a sone potentiometer. The listeners, seated in a sound-proofed booth, heard all stimuli binaurally through a pair of TDH-39 earphones mounted in MX-41/AR sockets. Prior to the loudness adjustments, masked and unmasked noise thresholds were measured individually by the method of limits. During each loudness matching session, the standard noise was presented two times at 7 to 10 sensation levels (SLs). The order of presentation was randomized, and it differed from listener to listener. Either the masked noise or the unmasked noise served as a standard, and the listener adjusted the loudness of the variable noise until it appeared as loud as the standard noise. The median threshold values of the group, which closely agreed with group geometric means, were used for the determination of the average SPLs of the noise.

Four to six listeners participated in each experimental series. Loudness matching between two equally wide bands of noise turned out to be a relatively simple task, so that as few as three listeners were needed to produce a stable mean result. Most of the loudness measurements were made with the noise between 40 and $110 \mathrm{~dB}$ SPL. This intensity region was emphasized because the determination of the masking effect of the tone in the vicinity of equal loudness between the tone and noise was of principal concern. For this purpose, rather than having the SPL of the masking tone adjusted individually, it was held constant from listener to listener. The SPLs of the masking tone ranged from 60 to $100 \mathrm{~dB}$.

A tone of constant SPL produced some inter-S threshold shift variability. (The inter-S difference in auditory sensitivity was actually within $8 \mathrm{~dB}$.) However, since no loudness judgments were made at very low sound intensities, this source of variability was not a problem. In computing the amount of masking produced by the tone, the median threshold shift of the group was used.

II. RESULTS AND DISCUSSION

A. Narrow, Octave, and Wide-Band Noise as Masked Signals

The results of the main experiment 


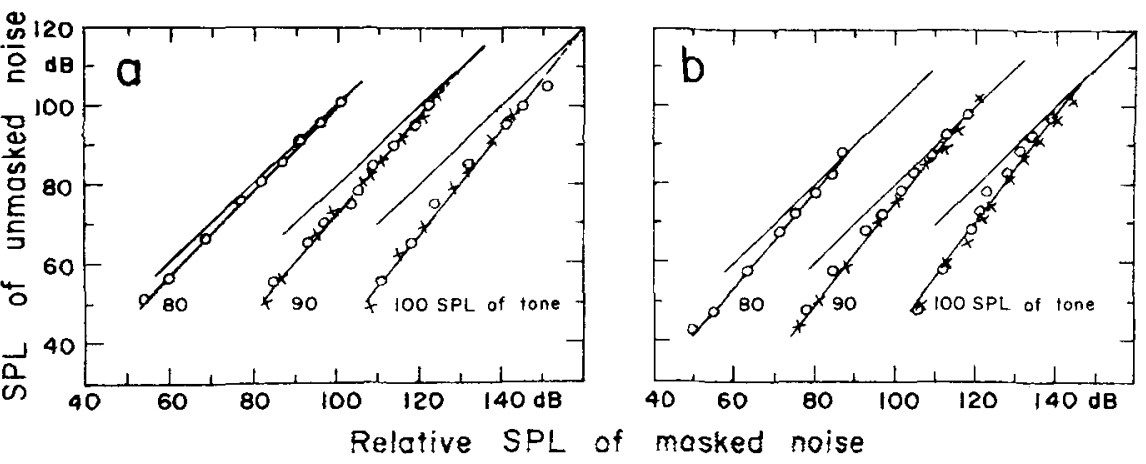

Fig. 1. (a) Loudness-matching functions obtained by equating in loudness a masked and unmasked band of noise. Noise bandwidth: $75-9,600 \mathrm{~Hz}$. Masking frequency: $1,000 \mathrm{~Hz}$. The crosses indicate group geometric means obtained by adjustment of the unmasked noise, and the unfilled circles indicate those obtained by adjustment of the masked noise. The parameter is the SPL of the 1,000-Hz masking tone. The numbers along the abscissa indicate the SPLs of the noise masked by the lowest intensity tone. To determine the SPLE of each successive function, subtract 20 or $40 \mathrm{~dB}$, respectively, from the abscissa values (b) Analogous to (a), except that the noise band was 600-1,200 $\mathrm{Hz}$ wide.

are plotted in Figs. 1 and 2. The data show the SPLs required for equal loudness between a band of noise in the presence of a pure tone and the same bandwidth of noise in the absence of the tone. Figures $1 \mathrm{a}$ and $1 \mathrm{~b}$ show the masking effect of the tone on broad-band and octave-band noise respectively. The effect of the tone on the $925-1,080-\mathrm{Hz}$ band is shown in Fig. 2. In the absence of masking, the median threshold value for the broad-band noise was $15 \mathrm{~dB}$ SPL. The median value for the octave-band noise was $8 \mathrm{~dB}$, and for the $925-1,080-\mathrm{Hz}$ band it was $6 \mathrm{~dB}$, which is also the unmasked 1,000-Hz threshold.

Most of the loudness-matching functions shown in Figs. 1 and 2 were determined using a counterbalanced experimental design; both the masked and unmasked noise bands served as standards. Each point is the geometric mean of 8 to 12 loudness judgments. The crosses indicate group means obtained by adjustment of the unmasked noise, and the unfilled circles indicate those obtained by adjustment of the masked noise. The parameter is the SPL of the masking tone.

It is apparent that adjustment of the masked or unmasked noise produces nearly the same result, indicating that the commonly found experimental biases (Stevens, 1961; Stevens \& Greenbaum, 1966) are very small. Therefore, to a first approximation, both series of data may be represented in log-log coordinates by a single straight line and thus by a single slope.

Over the range of intensities explored, the loudness-matching functions are power functions. Figure 1a shows that for wide-band noise as signal, the slope (exponent) increases from about 1.1 for a masking tone at $80 \mathrm{~dB}$ SPL to about 1.35 for a tone at $100 \mathrm{~dB}$ SPL. Correspondingly, the amount of masking at threshold, computed from the difference between masked and unmasked group thresholds, increases in 10-dB steps from about $10 \mathrm{~dB}$ to $30 \mathrm{~dB}$. In agreement with threshold data of Young and Wenner (1967), a 1,000-Hz tone below $90 \mathrm{~dB}$ SPL has practically no masking effect on a wide-band noise.

The masking effect of the tone is somewhat greater when the signal bandwidth is decreased to the width of an octave. The slopes of the segments in Fig. $1 \mathrm{~b}$ increase from about 1.25 for a masking tone at $80 \mathrm{~dB}$ SPL to about 1.45 for a tone at $100 \mathrm{~dB}$ SPL. Correspondingly, the amount of masking at threshold is somewhat greater; it increases in 10-dB steps from about $17 \mathrm{~dB}$ to $37 \mathrm{~dB}$. Wide- and octave-band data also show, contrary to the expectation of Stevens and Guirao (1967), that intense tones mask the noise somewhat, even when the SPL of the tone is less than the overall SPL of the noise. We shall see later on that it may be more appropriate to consider the effect of the noise energy within a narrow band surrounding the tone rather than the overall SPL.

While the difference between the masking effect of the tone on wideand octave-band signals is relatively small, decreasing the width of the signal to the $155-\mathrm{Hz}$ band produces a sharp increase in the masking effect of the tone. The slopes of the segments in Fig. 2 increase from about 2.25 for a masking tone at $60 \mathrm{~dB}$ SPL to about 2.6 for a tone at $90 \mathrm{~dB}$. Correspondingly, masking at threshold increases from about $33 \mathrm{~dB}$ to $56 \mathrm{~dB}$, but not in proportion to the SPL of the tone. The difference in masking effect of the tone on narrow- and wide-band signals is decreased somewhat when the data are examined with regard to a constant masking effect of the tone at threshold. Nevertheless, it is clear that the tone produces the strongest masking on the 155-Hz noise band.

The steepening of the $155-\mathrm{Hz}$ band functions may be attributed to the fact that the spread of excitation of the tone and noise is more nearly the same (Zwicker, 1958). Hence, the masking effect of the tone is increased. But is the increased amount of masking produced by the tone on the $925-1,080-\mathrm{Hz}$ noise band equivalent to the reduction in masking obtained by the noise on the tone when the bandwidth of the masking noise is decreased from 9,600 to $155 \mathrm{~Hz}$ ? The answer seems to be no. A narrow band of noise is a more effective masker than a pure tone centered on the noise. Perhaps the slopes of the matching functions for noise can tell us something more about this asymmetry.

Although the slopes of the segments in Fig. 2 tend to increase with increases in the amount of masking, they do not increase in proportion to the SPL of the masking tone According to Stevens (1966), the slopes ought to increase as a linear function of the SPL of the masking stimulus provided that the signal-to-noise ratios at the bottom and at the top of the steep segments are constant. It turns out, however, that for the $925-1,080-\mathrm{Hz}$ signal, the signal-to-noise ratio at the bottom of the functions increases with the SPL of the masking tone. For example, when the masking tone is at $60 \mathrm{~dB}$ SPL, the signal-to-noise ratio at threshold is close to $-20 \mathrm{~dB}$; when the masking tone is at $90 \mathrm{~dB}$ SPL, the

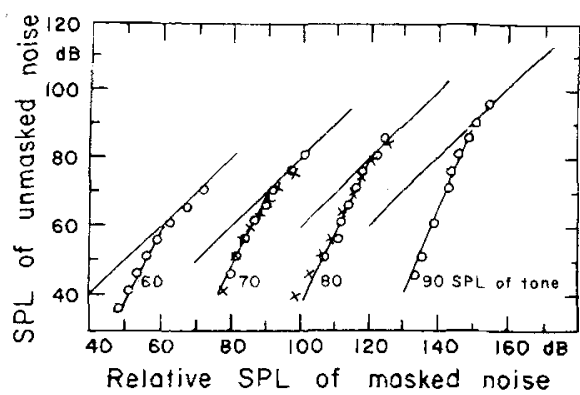

Fig. 2. Analogous to Fig. 1a, except that the noise band was 925-1,080 $\mathrm{Hz}$ wide. To determine the SPLs of the noise masked by the 90-dB SPL, tone, subtract $60 \mathrm{~dB}$ from the abscissa values. 

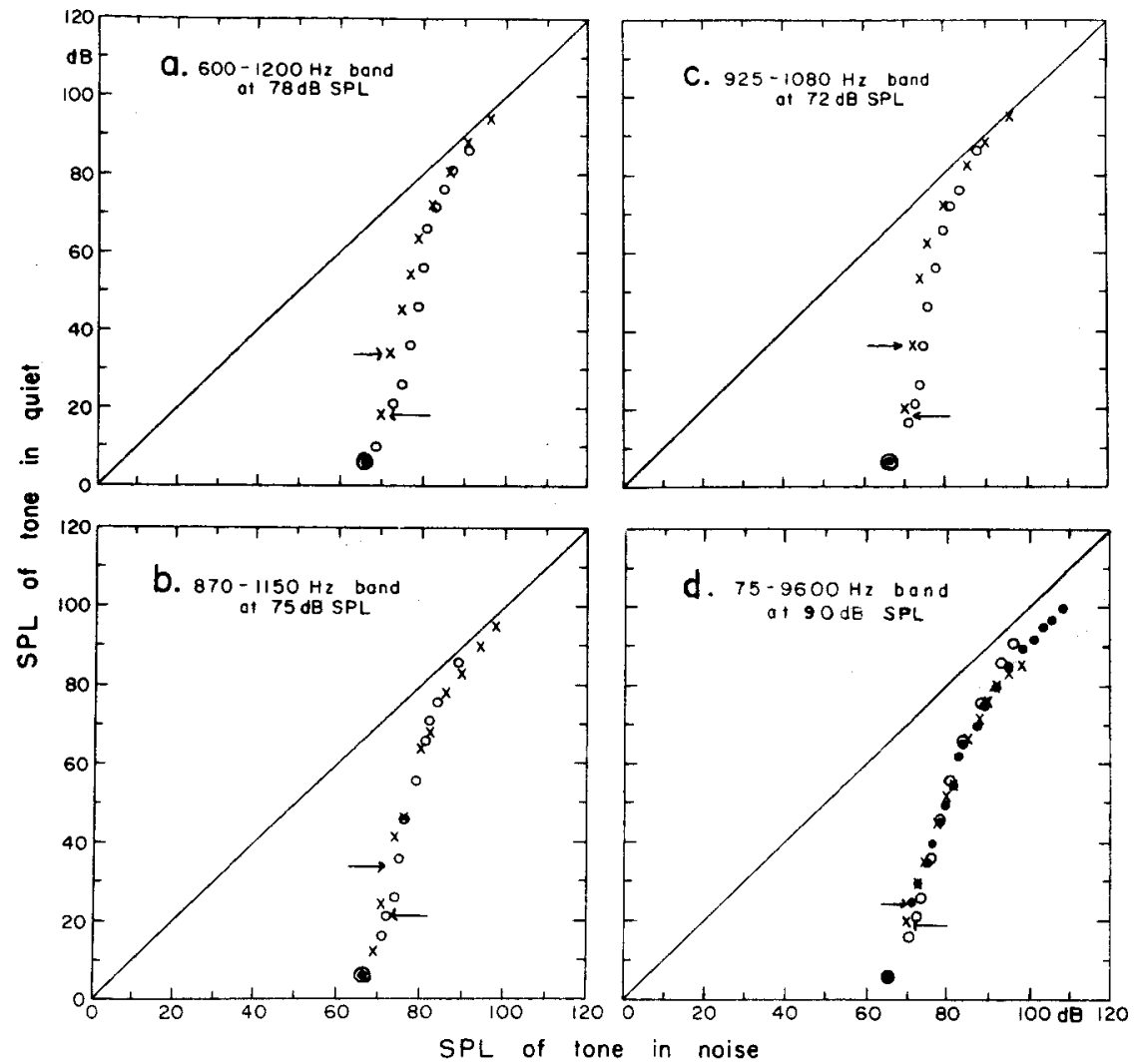

Fig. 3. (a) Loudness-balance data from Hellman (1970) obtained with a 600-1,200-Hz noise band that produced a $60-\mathrm{dB}$ threshold shift. Crosses: group geometric means of nine Os who adjusted the loudness of the unmasked tone. Unfilled circles: group geometric means of nine Os who adjusted the loudness of the masked tone. The arrows show where the overall energy of the $155-\mathrm{Hz}$ noise band that surrounds the tone and the energy of the tone is the same. The lowest point indicates the measured value of the masked threshold. (b) Analogous to (a), except that the noise band was $870-1,150 \mathrm{~Hz}$ wide. (c) Analogous to (a), except that the noise band was 925-1,080 $\mathrm{Hz}$ wide. (d) Analogous to (a), except that the noise band was $75-9,600 \mathrm{~Hz}$ wide. The crosses and filled circles are from the extensive wide-band measurements of Stevens and Guirao (1967).

ratio is close to $-30 \mathrm{~dB}$. The effectiveness of the tone as a masker seems to decrease with increasing SPL of the tone. These values do not seem to be a measurement fluctuation. They are in good agreement with earlier findings by Zwicker (1963) and are consistent with the masking patterns for tone and noise obtained by Ehmer (1959).

By contrast, when the narrow-band noise is used as a masker, the signal-to-noise ratio at the tone threshold is of the order of $-4 \mathrm{~dB}$ (Zwislocki, 1965), and according to Scharf $(1964,1971)$, this ratio does not change with increasing SPL of the noise. It is also of interest to note that individual listeners consistently report that the region of uncertainty in the vicinity of the threshold appears greater when the tone is used as a masker than when the masking stimulus is a band of noise. (This observation applies to both narrow and wide bands of noise.) Both as a masker and as a signal, noise is clearly dominant.

Let us now turn to the intercepts of the functions shown in Figs. 1 and 2, for they provide a more complete picture of the extent of the asymmetry. The intersection of the loudness-matching functions with the equal SPL line is clearer than when noise is the masker (Hellman \& Zwislocki, 1964; Hellman, 1970). The difference is probably due to the strong dominance of noise as a masker. Thus, when the tone masks the noise, the audibility of the tone, and hence its masking effect, is soon substantially reduced by the noise. At this point, it is almost trivial to match the two noises in loudness. The situation is not quite the same, however, for a noise masking a tone. When noise is the masker, even a noise as narrow as the $925-1,080-\mathrm{Hz}$ band, its presence remains very apparent at high intensities of the tone. Consistent with measures of the masked threshold, the effect of the noise tapers off more gradually than does the effect of the tone, i.e., the tone is more easily obliterated.

To a good approximation, the functions in Figs. 1 and 2 show that the tone no longer masks the noise (masked and unmasked noises are equally loud at the same overall SPL) when the energy of the tone and the energy in the $155-\mathrm{Hz}$ noise band surrounding the tone are equal. This means that for the 925-1,080-Hz band, masking ceases when the tone and noise are at the same overall SPL, while for the $600-1,200-\mathrm{Hz}$ band, the overall SPL of the noige is about $6 \mathrm{~dB}$ above that of the tone, and for the $75-9,600-\mathrm{Hz}$ band it is about $18 \mathrm{~dB}$ above that of the tone. The results obtained with the narrow-band signal are in accord with narrow-band measurements of Zwicker (1963) and with pure-tone data of Chocholle (1969).

Once again, we are confronted with the well-established ability of the ear (Fletcher, 1940; Zwicker, 1954) to filter out the energy contributed by frequencies of the noise that lie outside of the critical band for the tone. Only those noise frequencies that surround the tone contribute to its masking effect. Viewed in this way, the masked loudness data for noise agree with Stevens's (1966) suggestion that masking ceases when the masked and masking stimuli are equally intense. They also closely approximate the expectations of Zwicker (1963), since the point of equal intensity (energy) is near the masked threshold for the tone. Hence, for tones masking noise, the suggestions of Zwicker and of Stevens lead to the same result.

In the light of these findings, the question remains how best to interpret partial masking when the signal is a 1,000-Hz tone. Previous measurements by Zwicker (1963) and by Hellman (1970) using wide- and narrow-band noise to mask a $1,000-\mathrm{Hz}$ tone show that the point of equal energy between the tone and the band of noise that surrounds the tone occurs near the bottom of the masked loudness function for the tone. Figure 3 shows some examples of loudness-balance data for tone masked by narrow and wide bands of noise that produced a masking effect at a threshold of $60 \mathrm{~dB}$. Most of the data are from an earlier paper by Hellman (1970), but the crosses and filled circles in Fig. 3d are from the extensive wide-band measurements of Stevens and Guirao (1967). The arrows near the bottom of the functions indicate the point of equal energy between the masked tone and the $155-\mathrm{Hz}$ noise band that surrounds the tone.

Two ways to interpret these data appear possible. If we consider them 
on their face value in terms of the SPL of the masking stimuli, then the steepening of the functions in Fig. 3 above threshold is a measure of the partial masking produced by the noise on the tone. But, according to Figs. 1 and 2 , in the same intensity range the tone usually produces some masking effect on the noise. Because the masking effect of the tone is substantially increased when the masked signal is a narrow-band noise, the 9:5-1,080- $\mathrm{Hz}$ band as masker and signal provides the clearest example. Nevertheless, this statement also applies to the partial masking between strong tones and octave and wide bands of noise. Taken together, the data obtained for noise-masking tone and for tone-masking noise seem to suggest that mutual masking between tone and noise occurs along the steep portion of the masked loudness function.

However, another approach appears plausible. Rather than compare the masking effects of noise and tone at the same overall SPLs, suppose we compare the effects of those stimuli that produce equal amounts of masking at threshold. When this is done, the symmetry of the masked loudness functions for tone and noise is markedly improved. For wide-band noise, it means that we must, for example, compare the masking effect of a 90-dB SPL noise with that of a 130-dB SPL tone. Such a comparison is obviously impractical. Hence, for practical purposes, this approach is restricted to narrow-band noise.

Figure 4 provides a concrete illustration. The linear segments, taken from Fig. 2, show the partial masking produced by a $1,000-\mathrm{Hz}$ tone at SPLs of 70 and $90 \mathrm{~dB}$ on the $925-1,080-\mathrm{Hz}$ noise band, and the data from Fig. 3c show the effect of the 72-dB SPL noise on the tone. The lowest points indicate the measured values of the masked threshold, and the arrows show where theoverall energy of the masking noise and the energy of the tone in noise is the same. Surprisingly, with respect to both position and slope, the masking effect of the 925-1,080-Hz band at $72 \mathrm{~dB}$ SPL closely resembles the effect of the tone at $90 \mathrm{~dB}$ SPL. In fact, as a masker, the narrow-band noise produces a slightly steeper function, but this result can be accounted for by the observation that the masking effect of the noise at threshold is somewhat larger than that of the tone.

From this point of view, the behavior of the 72-dB SPL noise is like that of the 90-dB SPL tone. Although the masked loudness data for tone do not produce a clearly defined intersection with the equal SPL line, it seems reasonable to suggest that

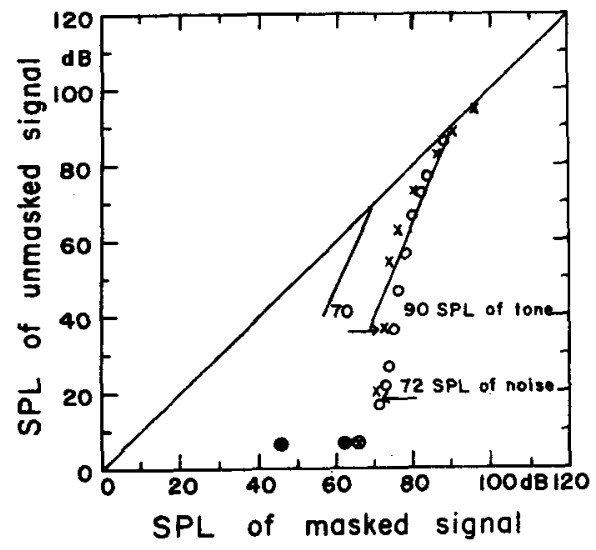

Fig. 4. Comparison between the makking effects of a $1,000-\mathrm{Hz}$ tone and those of a $925-1,080-\mathrm{Hz}$ noise band at 72 dB SPL. The linear segments that show the masking effect of the tone at SPLs of 70 and $90 \mathrm{~dB}$ are from Fig. 2 , and the loudness-balance data for tone are from Fig. 3c. The lowest points indicate the measured values of the masked threshold, and the arrows show where the overall energy of the noise and the energy of the tone in noise are the same.

masking by the noise ceases when the SPL of the tone is about $90 \mathrm{~dB}$. Below $90 \mathrm{~dB}$, the masked loudness function for the tone is determined by the effect of the noise. Similarly, below a SPL of the noise of $90 \mathrm{~dB}$, the masked loudness function for noise is determined by the effect of the tone. This inverse relationship holds if we assume that noise masks the tone until the effective energy of both the noise and the tone is the same.

In another example, despite the large disparity in overall SPLs, the 100-dB tone as masker of the $600-1,200-\mathrm{Hz}$ band (see Fig. 1b) produces results in excellent agreement at medium and high sound intensities with those of Hellman and Zwislocki (1964), who used a $600-1,200-\mathrm{Hz}$ noise at $57 \mathrm{~dB}$ SPL to mask the tone. For both tone and noise as maskers, the threshold shift was about $40 \mathrm{~dB}$. While two examples cannot constitute a proof, they do provide an interesting extension of Stevens's (1966) interpretation of masking. An additional study, especially designed to measure the loudness of masked noise at low sound intensities, is needed. Whether the masked loudness functions for noise, like the masked loudness functions for tone (Hellman \& Zwislocki, 1964; Chocholle \& Greenbaum, 1966; Chocholle, 1969; Hellman, 1970), also curve at near-threshold intensities is an intriguing question that remains to be answered.
B. Critical and Subcritical Noise Bands as Masked Signals

The asymmetry of masking between the $925-1,080-\mathrm{Hz}$ band and the $1,000-\mathrm{Hz}$ tone is puzzling, for this noise is approximately the critical bandwidth at $1,000 \mathrm{~Hz}$ (Zwicker, 1961). Measurements of loudness summation, using multitone complexes or narrow and wide bands of noise as signals yield the same estimates of the critical bandwidth at $1,000 \mathrm{~Hz}$ (Scharf, 1970). Yet, masked threshold data have persistently shown that the amount of masking produced by a narrow band of noise is greater than that obtained with a pure tone at the center frequency of the noise (Egan \& Hake, 1950; Hirsh et al, 1950; Ehmer, 1959; Zwicker, 1963; Young \& Wenner, 1967). It is evident from the preceding section that narrow-band noise also dominates as a partiai masker. The difference between the effects of noise and tone is of the order of $20 \mathrm{~dB}$. The question is, why?

The experiments to be described in this section attempt to deal with this question. Empirically, we can ask if the masking effect of the tone is increased if the bandwidth of the masked signal is reduced to a subcritical width. For this purpose, two additional loudness matching experiments, consisting of two sessions each, were performed. In both experiments, the SPL of a $1,400-\mathrm{Hz}$ tone was held constant at $70 \mathrm{~dB}$, but in one the masked noise band was $1,280-1,480 \mathrm{~Hz}$ wide and in the other it was $1,350-1,450 \mathrm{~Hz}$ wide.

Figure 5a shows the results obtained with the $1,280-1,480-\mathrm{Hz}$ band, and Fig. 5b shows those obtained with the 1,350-1,450-Hz band. Each point, except the lowest one, is the geometric mean of 10 to 12 loudness judgments determined by five to six listeners. The crosses indicate group geometric means obtained by adjustment of the unmasked noise, the unfilled circles indicate those obtained by adjustment of the masked noise, and the lowest point indicates the average value of the masked threshold. For comparison Fig. 5a also shows the averages (filled circles) obtained with the 925-1,080-Hz signal and a masking tone of $1,000-\mathrm{Hz}$ at $70 \mathrm{~dB}$ SPL. The small change in masker frequency from 1,000 to $1,400 \mathrm{~Hz}$ does not alter the results; at both frequencies the noise band was approximately one critical band wide at that frequency (Zwicker, 1961).

According to Fig. 5, adjustment of the masked or unmasked noise produces almost the same result. Thus, as in Figs. 1 and 2, a single linear function provides a reasonably good description of the data. Between SPIs of about 20 and $70 \mathrm{~dB}$, the function 


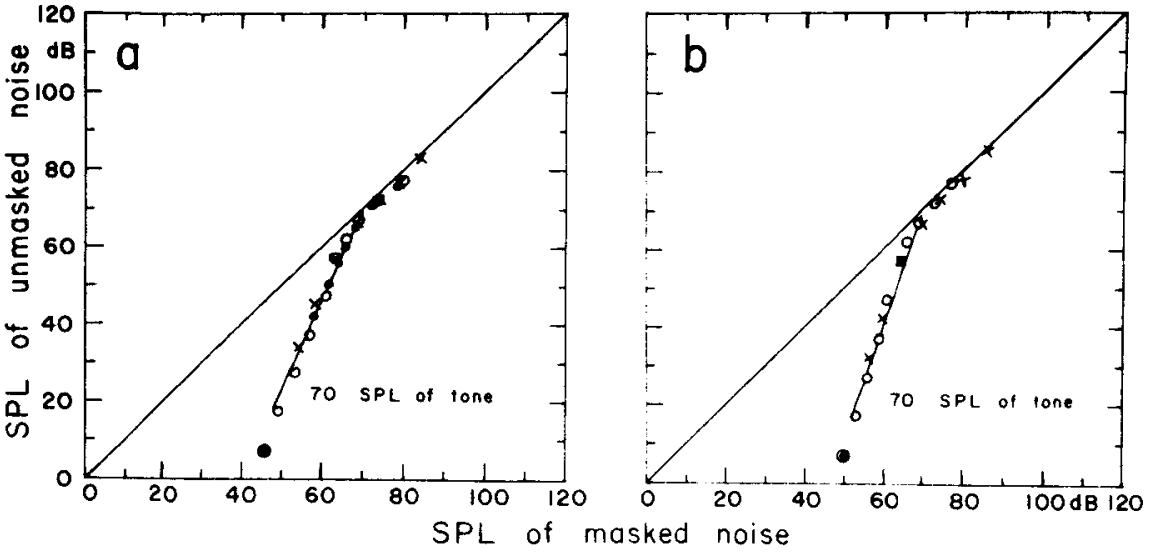

Fi.g 5. (a) Loudness-balance data obtained for a 1,280-1,480-Hz noise band masked by a 1,400-Hz tone. The crosses indicate group geometric means obtained by adjustment of the unmasked noise, the unfilled circles indicate those obtained by adjustment of the masked noise, and the lowest point indicates the measured value of the masked threshold. The filled circles are average values from Fig. 2, obtained with the 925-1,080-Hz signal and a masking tone of 1,000-Hz at 70 dB SPL. (b) Analogous to (a), except that the noise band was 1,350-1,450 $\mathrm{Hz}$ wide.

produced by the $1,350-1,450-\mathrm{Hz}$ band is steeper than the one produced by the 1,280-1,480- $\mathrm{Hz}$ band. The slope of the line in Fig. 5a is about 2.4 and that of the line in Fig. 5b is about 3.0. For the subcritical noise band, like the critical band noise, masking by the tone practically ceases when the overall energy of the noise and tone is the same.

Recent measurements by Scharf (1971b) show that the spread of masking of a subcritical band of noise is narrower than that produced by an equally intense critical band of noise. This result could account for the increased masking effect of the tone on the 1,350-1,450- $\mathrm{Hz}$ band. Figure 5 shows that the increase at threshold is of the order of $4 \mathrm{~dB}$. Nevertheless, while a reduction in noise bandwidth improves the symmetry of the masked loudness functions for tone and noise, most of the 20-dB difference between the masking effects of tone and noise remains.

The data suggest that, although a pure tone and an equally intense narrow-band noise are equally loud, this result is not related to the masking ability of tone and noise. An interpretation of narrow-band results is complicated by the fact that a reduction in bandwidth is accompanied by a decrease in the rate of intensity fluctuations (Stevens, 1956). Bos and de Boer (1966) point out that the slow rate of intensity fluctuations inherent in the structure of narrow-band noise increases its ability to mask. Masking contours by Scharf (1971b) provide some support for the work of Bos and de Boer. They show that the peak masking produced by a subcritical band of noise is greater than that produced by an equally intense critical band of noise. Interestingly, masked threshold data by Young and Wenner (1967) indicate that the 20-dB difference between the masking effect of a tone and a narrow critical band of noise disappears when the pure tone is replaced by a tone that is frequency modulated at a rate of $25 / \mathrm{Hz}$. The use of a frequency-modulated tone as a partial masker deserves further study.

\section{SUMMARY OF RESULTS}

The steepness of the loudness-matching functions for noise masked by tone depends on both the SPL of the masking tone and the bandwidth of the masked noise. When the noise bandwidth is held constant and the SPL of the masking tone is increased, the functions tend to steepen. By contrast, when the SPL of the masking tone is held constant but the noise bandwidth is increased, they tend to become flatter. The effect of the tone on a noise band $925-1,080 \mathrm{~Hz}$ wide is greater than its effect on either an octave- or wide-band noise. However, it is considerably smaller than the masking effect of the 925-1,080-Hz band on the tone. Halving the bandwidth of the noise improves the symmetry of masking at threshold by about $4 \mathrm{~dB}$, but a difference at high intensities of close to $20 \mathrm{~dB}$ between the masking effects of an equally intense tone and noise remains. It is possible that the slow rate of intensity fluctuations inherent in narrow-band noise accounts for most of the difference. The data also suggest that, for both noise and tone, masking ceases when the effective energy of the masked and masking stimuli is the same.

\section{REFERENCES}

BOS, C. E., \& de BOER, E. Making and discrimination. Journal of the Acoustical Society of America, 1966, 39, 708-715. CHOCHOLLE, R. Quelques expérlences sur l'effet de masque partiel. International Audiology, 1969, 8, 125-130.

CHOCHOLLE, R., \& GREENBAUM, H. B. La sonie de sons purs partiellement masqués. Etude comparative par une méthode d'Gralisation et par la méthode des temps de réaction. Joumal de Psychologie, 1966, 4, 385-414.

EGAN, J. P., \& HAKE, H. W. On the masking pattern of a simple auditory timulus. Journal of the Acoustical Society of America, 1950, 22, 622-630.

EHMER, R. Masking by tones vs noise bands. Journal of the Acoustical Society of America, $1959,31,1253-1256$.

FLETCHER, H. Auditory pattems. Review of Modern Physics, 1940, 12, 47-65.

HELIMAN, R. P. Effect of noise bandwidth on the loudness of a $1000-\mathrm{Hz}$ tone. Joumal of the Acoustical Society of America, 1970, 48, 500-504.

HELLMAN, R. P. ZWISLOCKI, J. J. Loudness function of a 1000-cps tone in the presence of a masking noise. Journal of the Acoustical Society of America. $1964,36,1618-1627$.

HIRSH, I. J., ROSENBLITH, W. A. \& WARD, W. D. The masking of clicks by pure tones and bands of noise. Journal of the Acoustical Society of America, 1950, 22, 631-637.

SCHARF, B. Partial masking. Acustica, 1964, 14, 17-23.

SCHARF, B. Critical bands. In: Foundations of modern auditory theory. Vol. I. New York: Academic Press, 1970. Pp. 157-202

SCHARF, B. Fundamentals of auditory masking. International Audiology, 1971a, $10,30-40$.

SCHARF, B. Pattems of partial masking Seventh International Congress on Acoustics, Budapest, 1971b (in press).

STEVENS, S. S. Calculation of the loudness of complex noise. Journal of the Acoustical Society of America, 1956, 28, 807-832.

STEVENS, $S$. S. The psychophysics of sensory function. In $\mathrm{W}$. A. Rosenblith (Ed.), Sensory communication. Cambridge, Mass: M.I.T. Press; New Yokk: Wiley, 1961. Pp. 1-33.

STEVENS. S. S. Power-group transformations under glare, masking and recruitment. Journal of the Acoustical Society of America, 1966, 39, 725-735. STEVENS, S. S., \& GREENBAUM, H. Regression effects in psychophysical judgments. Perception \& Psychophysics. $1966,1,439-446$.

STEVENS, S. S., \& GUIRAO, M. Loudness functions under inhibition. Perception \& Psychophysics, 1967, 2, 459-465.

Y OUNG, I. M., \& WENNER, C. H. Maskin of white noise by pure tone, frequency-modulated tone, and narrow-band noise. Joumal of the Acoustical Society of America, 1967, 41, $700-706$.

ZWICKER, E. Die Verdekung von Schmalbandgereräuschen durch Sinustone. Acustica, 1954, 4, 415-420.

ZWICKER, E. Ubex psychologische und methodische Grundlagen dex Lautheit. Acustica, 1958, 8, 237-258.

ZWICKER, E. Subdivision of the audible frequency range into critical bands. (Frequenzgruppen). Journal of the Acoustical Society of America, 1961, 33, 248.

ZWICKER, E. Über die Lautheit von ungedrosselten and gedrosselten Schallen. Acustica, 1963, 13, 194-211. 
ZWICKER, E., FLOTTORP, G., \& STEVENS, S. S. Critical bandwidth in loudness summation. Journal of the
5 48-557.

ZWISLOCKI, J. J. Analysis of some auditory characteristics. In $R$. D. Luce, $R$. R. Bush, and E. Galanter (Eds.),
Handbook of mathematical psychology. Vol. 3. New York: Wiley, x965. Pp. 1-97.

(Received for publication June 1, 1971.) 